\title{
Neonatal colonization of mice with LGG promotes intestinal development and decreases susceptibility to colitis in adulthood
}

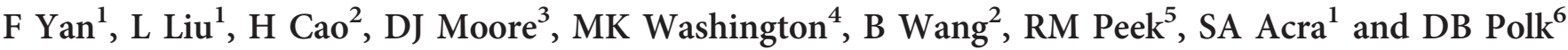

Development of the intestinal microbiota during early life serves as a key regulatory stage in establishing the hostmicrobial relationship. This symbiotic relationship contributes to developing host immunity and maintaining health throughout the life span. This study was to develop an approach to colonize conventionally raised mice with a model probiotic bacterium, Lactobacillus rhamnosus GG (LGG), and to determine the effects of LGG colonization on intestinal development and prevention of colitis in adulthood. LGG colonization in conventionally raised was established by administering LGG to pregnant mice starting at gestational day 18 and pups at postnatal days $1-5$. LGG colonization promoted bodyweight gain and increased diversity and richness of the colonic mucosa-associated microbiota before weaning. Intestinal epithelial cell proliferation, differentiation, tight junction formation, and mucosal IgA production were all significantly enhanced in LGG-colonized mice. Adult mice colonized with LGG showed increased IgA production and decreased susceptibility to intestinal injury and inflammation induced in the dextran sodium sulfate model of colitis. Thus, neonatal colonization of mice with LGG enhances intestinal functional maturation and IgA production and confers lifelong health consequences on protection from intestinal injury and inflammation. This strategy might be applied for benefiting health in the host.

\section{INTRODUCTION}

Colonization of the intestinal microbiota in humans, occurring in utero, ${ }^{1}$ is significantly affected by microbial exposures at birth. $^{2}$ Post-natal colonization is a dynamic and genetically controlled process, leading to establishing a symbiotic relationship between the host and the microbiota. ${ }^{3}$ This relationship provides an ecosystem that includes a nutrient-rich environment for bacteria, while conferring to the host important contributions, including nutrient metabolism, functional maturation, and the development of immunity. ${ }^{4}$

Clinical studies have revealed that interruption of the hostmicrobial relationship leads to several diseases, such as inflammatory bowel disease, consisting of ulcerative colitis and Crohn's disease. ${ }^{5-7}$ Antibiotic treatment has been shown to alter the gut microbiome in humans, ${ }^{8}$ and the antibiotic exposure in childhood is associated with an increased risk of developing inflammatory bowel disease. ${ }^{9}$ Animal studies have demonstrated several defects in development of intestinal morphology, motility, epithelial proliferation and differentiation, and the immune system in germ-free animals. ${ }^{10}$ These defects in germ-free animals can be restored through monoassociation. ${ }^{11}$ Altering the intestinal microbiota during early development by antibiotic treatment has long-term metabolic consequences even when the microbiota returns to a normal population structure after the cessation of antibiotics. ${ }^{12}$ These findings emphasize the important contribution of the intestinal microbiota to intestinal homeostasis during development and throughout the life span.

Probiotics have shown clinical benefits on humans of different ages. For example, preliminary evidence suggests

\footnotetext{
${ }^{1}$ Division of Gastroenterology, Hepatology and Nutrition, Department of Pediatrics, Vanderbilt University Medical Center, Nashville, Tennessee, USA. ${ }^{2}$ Department of Gastroenterology and Hepatology, General Hospital, Tianjin Medical University, Tianjin, PR China. ${ }^{3}$ Division of Endocrinology, Department of Pediatrics, Vanderbilt University Medical Center, Nashville, Tennessee, USA. ${ }^{4}$ Department of Pathology, Microbiology and Immunology, Vanderbilt University Medical Center, Nashville, Tennessee, USA. ${ }^{5}$ Division of Gastroenterology, Departments of Medicine and Cancer Biology, Vanderbilt University Medical Center, Nashville, Tennessee, USA and ${ }^{6}$ Departments of Pediatrics and Biochemistry and Molecular Biology, University of Southern California and Saban Research Institute of Children's Hospital Los Angeles, Los Angeles, California, USA. Correspondence: F Yan or DB Polk (fang.yan@vanderbilt.edu or dbpolk@chla.usc.edu)
} 
that probiotics have efficacy for treatment of infants and young children with infectious diarrhea ${ }^{13,14}$ and prevention of antibiotic-associated diarrhea. ${ }^{14}$ However, a number of metaanalyses vary in their conclusions on the effectiveness of probiotics on disease prevention and treatment. ${ }^{15}$ Therefore, hypothesis-driven clinical trials using optimal strains, dose, and delivery and treatment protocols are needed.

Several animal studies have suggested that short-term treatment of probiotics, such as $\mathrm{LGG}^{16}{ }^{16}$ L. reuteri, ${ }^{17}$ and E. coli Nissel 1917, ${ }^{18}$ enhanced proliferation, migration, differentiation, barrier function formation, and immune defense in intestinal epithelial cells in mice. These findings underscore the importance of identifying the effects of probiotics on intestinal development and disease prevention in the later stage of life.

LGG, a naturally occurring Gram-positive bacterium, was originally isolated from the healthy human intestine. LGG has powerful adhesive properties for intestinal cells. ${ }^{19}$ Perinatal administration of LGG, $L$ acidophilus La-5, and Bifidobacterium animalis subsp. lactis $B b-12$ to mothers led to colonization of LGG, but not the other two bacteria, in the children at 10 days and at 3 months of age, which indicates that LGG has high ability to transfer from the mother to the child and colonize in the child. ${ }^{20}$ LGG has been widely used as a nutritional supplement. Clinical studies have demonstrated that perinatal LGG supplementation to mothers reduced the incidence of eczema at 2, 4, and 7 years of life in at-risk children. ${ }^{21-23}$ Feeding infants with formulas supplemented with LGG was well tolerated and supported growth. ${ }^{24}$ LGG has also shown beneficial effects on treating and/or preventing several disorders, including diarrhea and atopic dermatitis. ${ }^{25}$ To further understand the mechanisms underlying the effects of LGG on disease prevention and treatment, we have demonstrated that LGG and the LGG-derived protein, p40, prevented cytokineinduced apoptosis, preserved barrier function, and upregulated mucin production in cultured intestinal epithelial cells and in tissue culture models, thereby prevention and treatment of experimental colitis. ${ }^{26-28}$

Here, we colonized conventionally raised neonatal mice with LGG and provided evidence that LGG colonization promoted growth, epithelial proliferation, differentiation, tight junction formation, $\operatorname{IgA}$ production, and maturation of the intestinal microbiota during development and decreased susceptibility to intestinal injury and colitis in adult mice. These results support the efficacy of administration of LGG at early life for enhancing intestinal functional maturation and long-term health consequences in adults.

\section{RESULTS}

\section{Generation of an approach for colonization of conventionally raised mice with LGG}

The evidence that the microbial exposure at birth shapes the acquisition and structure of the initial microbiota in newborns ${ }^{2}$ supports the importance of early exposure of microbes for colonization. Furthermore, studies in germ-free mice showed that there was a time window for colonization by a

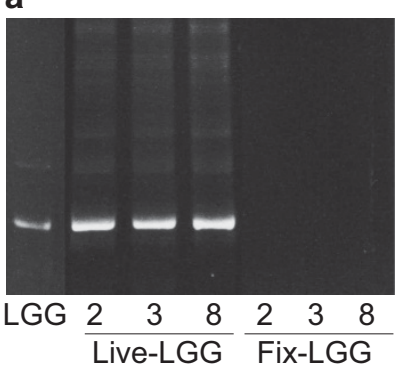

Age (week)

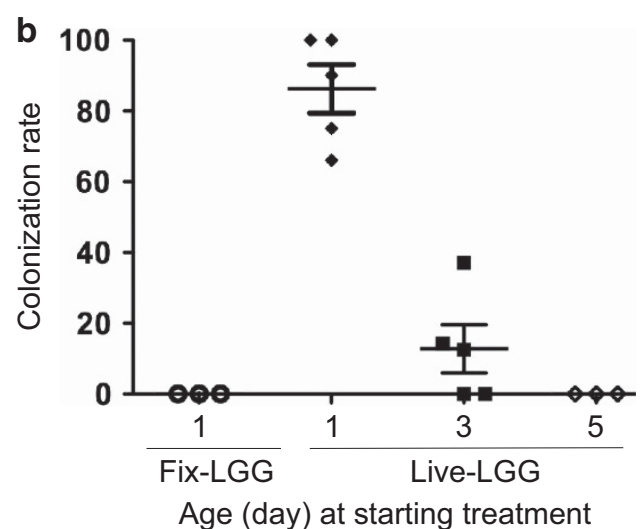

Figure 1 Detection of Lactobacillus rhamnosus GG (LGG) in mouse fecal bacteria. Fecal bacteria collected from mice at indicated ages with live LGG (Live-LGG) and glutaraldehyde-fixed LGG (Fix-LGG) treatment from postnatal days 1 to 5 were cultured in deMan, Rogosa and Sharpe broth. DNA was isolated from cultured bacteria for PCR analysis to amplify L. rhamnosus-specific 16S rRNA gene. PCR products were separated on denaturing gradient gel electrophoresis (DGGE) to determine DNA migration profiles (a). Feces of Live-LGG and Fix-LGG treatment were prepared from the same mouse at indicated ages. First lane contains the ladder composed of the PCR product from LGG. LGG colonization was defined as detection of LGG in fecal bacteria. The colonization rate of individual litter was determined by the percentage of mice with LGG colonization in the total number of mice from the same litter. The colonization rate of individual litter detected in 3-week-old mice treated with Live-LGG and Fix-LGG starting at indicated age (postnatal day) for 5 days is shown (b).

cecal contents. ${ }^{29}$ Therefore, we first generated an approach for colonization of conventionally raised mice with LGG.

We treated wt pregnant mice with live LGG (Live-LGG) from gestation day 18 to delivery and newborn mice starting at postnatal days 1, 3, and 5 for 5 days. Glutaraldehyde-fixed LGG (Fix-LGG) was used as a non-colonization control in this study. Colonization of LGG was defined by recovery of LGG from cultured mouse fecal bacteria by DNA fingerprint analysis. ${ }^{30,31}$ PCR analysis was performed to amplify $16 \mathrm{~S}$ rRNA bacterial genes using specific primers for Lactobacillus rhamnosus. ${ }^{31}$ DNA from LGG was used as a marker for the DNA fingerprint analysis. The migration profile of PCR amplified products from cultured fecal bacteria of 2-, 3-, and 8-week-old mice treated with Live-LGG from postnatal days 1 to 5 showed the specific band for LGG (Figure 1a). The PCR products were further analyzed by direct sequencing to verify the presence of LGG in LGG-colonized mice. In mice treated with Live-LGG from postnatal days 1 to $5,100 \%$ of mice with LGG detected in feces 
at 2- and 3-week old had LGG in feces at 8 weeks old, which indicates that neonatal LGG colonization can persist to adulthood. We did not find the LGG-specific PCR band from mice treated with Fix-LGG for the same period of time (Figure 1a). We also verified the presence of LGG in mouse feces by quantitative PCR analysis using LGG-specific primers, as previously reported. ${ }^{32}$ Results from quantitative PCR analysis showed that the amount of LGG in feces from LGG-colonized mice identified by the DNA fingerprint analysis was significantly higher than that in mice without LGG colonization (Supplementary Figure 1 online).

The colonization rate, which was detected in individual litter at 3 weeks old, was $70-100 \%, 0-40 \%$, and 0 , in mice receiving Live-LGG from postnatal days 1 to 5,3 to 8 , and 5 to 10 , respectively (Figure $\mathbf{1 b}$ ). Thus, these results suggest that colonization of conventionally raised mice with LGG is age dependent. Mice treated with Live-LGG and Fix-LGG from postnatal days 1 to 5 were used as the LGG colonization model and non-colonization control, respectively, in this study.

\section{LGG colonization promotes growth, intestinal epithelial cell proliferation, differentiation, and tight junction formation during development in mice}

As it has been reported that feeding infants with formulas supplemented with LGG supported growth ${ }^{24}$ and affected development of the intestinal microbiota, ${ }^{33}$ we next examined whether LGG colonization in mice promoted growth and maturation of the intestinal microbiota during development.

Mice with LGG colonization showed increased bodyweight gain from 1 to 4 weeks of age, but no differences were found in older mice, as compared with non-colonized mice (Figure 2a). Consistent with this result, LGG-colonized mice exhibited increased length of villi and depth of crypts at 2 weeks old (Figure 2b,c). However, we did not find any difference in these parameters between LGG-colonized and non-colonized mice at 8 weeks old (data not shown). These results indicate a beneficial effect of LGG colonization on intestinal growth during early life.

Functional maturation of the gastrointestinal tract completes by the third postnatal week in rodents. Thus, we examined the effects of LGG colonization on regulation of intestinal epithelial cell proliferation, differentiation, and barrier function formation in 2-week-old pups. Intestinal tissue sections were immunostained using antibodies against Ki67 and MUC2 to detect proliferation and differentiation, respectively. The numbers of Ki67 (Figure 3a) and MUC2-positive cells (Figure 3b) were increased significantly in pups with LGG colonization. In addition, Muc2 gene expression in colonic epithelial cells isolated from pups with LGG colonization was significantly increased ( $2.1 \pm 0.5$ fold, Figure $3 \mathbf{c})$, as compared with that in non-colonized mice.

Although development of barrier function occurs before birth, there is ongoing maturation postnatally. We have reported that LGG-derived p40 prevents cytokine and hydrogen peroxide disruption of tight junctions in vivo and in vitro. ${ }^{27,34,35}$ Thus, we next assessed the tight junction formation in LGG-colonized mice using three approaches. The in vivo permeability assay was performed to examine barrier function by measuring the serum FITC-dextran level after injection of FITC-dextran into the small intestine. The serum FITC-dextran in LGG-colonized mice was significantly lower than that in non-colonized mice (Figure 3d), which indicates maturation of barrier function by LGG colonization. LGG treatment has been found to increase mRNA expression of a tight junction protein, claudin 3 (CLDN3) in neonatal mice. ${ }^{16}$ We found that mRNA expression of CLDN3 was increased in neonatal mice with LGG colonization (Figure 3e). Immunostaining of $\mathrm{ZO}-1$ showed that LGG colonization increased ZO-1 membrane localization in the ileum (Figure 3f). These data suggest that LGG colonization promotes tight junction formation in neonatal mice.

In total, these results indicate that LGG colonization has the potential effects on promoting intestinal functional maturation through promoting proliferation, differentiation, and tight junction formation during development in mice.

\section{LGG colonization enhances IgA production during development and in adulthood}

IgA in the gastrointestinal tract has an important role in maintenance of the homeostatic community of commensal bacteria and protects the host against pathogenic infections. ${ }^{36}$ Probiotics have been reported to strengthen the immune response by increasing the production of IgA. ${ }^{37,38}$ Thus, we examined the effects of LGG colonization on IgA production. The IgA level in feces was measured by ELISA. Fecal IgA levels in 3- and 8-week-old mice with LGG colonization were significantly higher than those of age-matched non-colonized mice (Figure 4a). Immunostaining showed that LGG colonization increased the number of IgA-expressing cells in lamina propria of the small intestine (Figure $4 \mathbf{b}$ ). The number of fecal Lactobacillus colonies was examined by culturing fecal bacteria on deMan, Rogosa and Sharpe agar. The fecal sIgA level directly correlated with the number of Lactobacillus in feces in 3- and 8-week-old mice with LGG colonization (Figure 4c), which further suggests that LGG colonization has a role in regulation of IgA production in the intestine.

\section{LGG colonization benefits maturation of the intestinal microbiota during development}

We next studied the impact of evolution of LGG colonization on development of the intestinal microbiota. Community profiling of the colonic mucosally-adherent bacteria isolated from 3 and cecal bacteria from 8 -week-old mice was assessed by pyrosequencing of amplified bacterial $16 \mathrm{~S}$ rDNA. LGG colonization increased Shannon diversity and Chaol richness in 3-week-old mice (Figure 5a,b). There were no differences of richness and diversity of microbiota in between LGG-colonized and non-colonized mice in 8-week-old mice (Supplementary Figures $2 \mathbf{A}$ and $\mathbf{B}$ ). It should be noted that the diversity and richness of the intestinal microbiota in 3-week-old LGGcolonized mice showed similarity to that of 8-week-old mice with and without LGG colonization. 

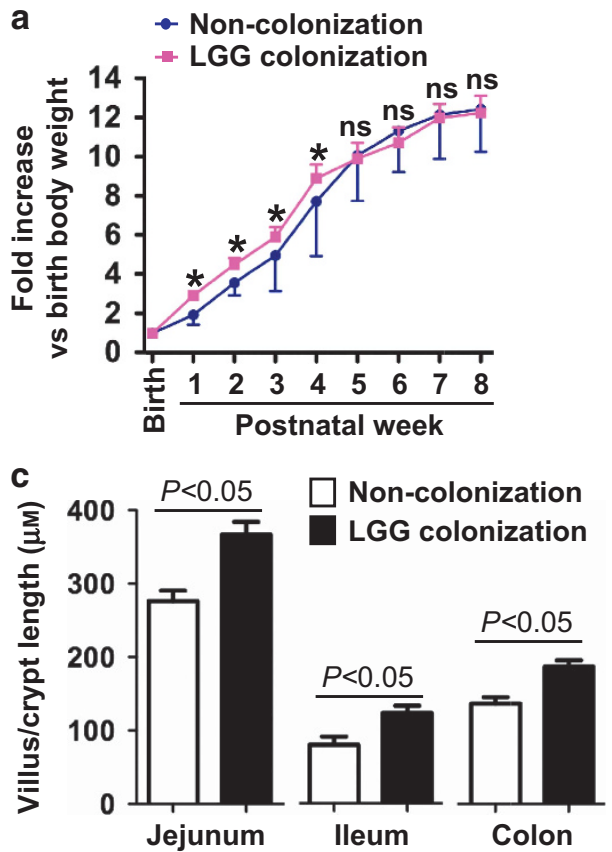

b

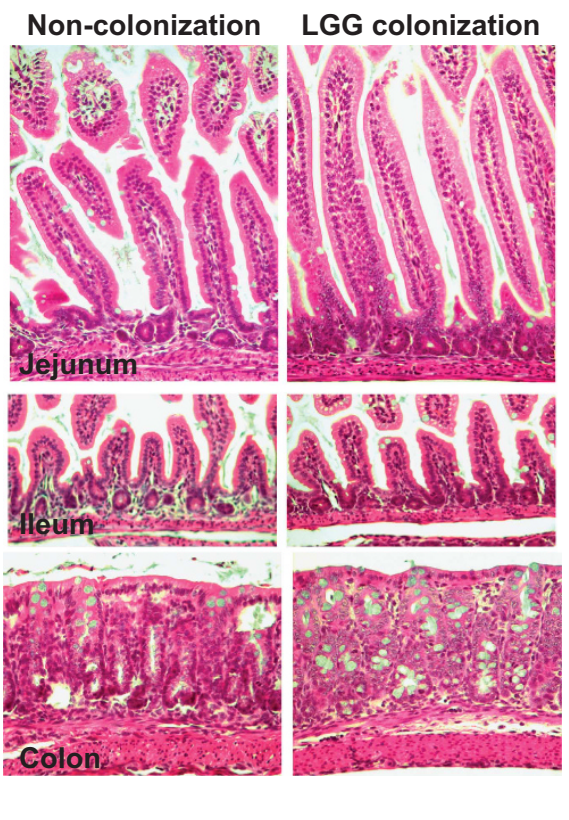

Figure 2 Colonization of conventionally raised mice with Lactobacillus rhamnosus GG (LGG) accelerates growth and development of intestinal microbiota before weaning. Mice were treated with live LGG from postnatal days 1 to day 5, and colonization was confirmed by detecting LGG in fecal bacteria, as described in Figure 1. Fixed LGG was used to treat mice as a non-colonization control. Bodyweight was recorded. Bodyweight at birth was set as 1 (a). ${ }^{*} P<0.05$ compared with the control group at the same age. Indicated intestinal tissues from 2-week-old mice were prepared for H\&E staining (b), and the length of villus and the depth of crypt were measured in at least 50 villi and crypts/mouse (c). In a-c, non-colonization: $n=17$, LGG colonization: $n=15$.

The functional maturation of the intestinal microbiome in humans includes compositional shifts in the abundances of major microbial taxa, with the intestinal microbiota of the young resembling that of the adult by approximately 3 years of age. ${ }^{39}$ To gain greater insight into the effects of LGG colonization on maturation of mouse intestinal microbiome, we analyzed the abundance of specific taxa of the microbiota for comparing the microbial composition in 3-week-old mice with that in 8-week-old mice with and without LGG colonization. Genus-level analysis showed that major taxonomic groups with abundance from high to low were Bacteroides, Lactobacillus, Clostridium, and Helicobacter in 3-week-old non-colonized mice (Figure 5c), which were shifted to Lactobacillus, Clostridium, and Bacteroides in 8-week-old non-colonized mice (Supplementary Figure 2C). Thus, establishment of the intestinal microbiota in adult mice is a dynamic process, with significant change of the microbial compositions, such as a decrease in Bacteroides in adult mice.

LGG colonization stimulated a significant expansion of Lactobacillus and Clostridium and contraction of Bacteroides and Helicobacter in 3-week-old mice (Figure 5c). Although LGG colonization in 8-week-old mice caused a significant expansion of Lactobacillus, Bacteroides, and Blautia, and contraction of Parasutteralla and Aldercreutzia (Supplementary Figure 2C), the pattern of abundance of the major bacteria was not change. It should be noted that LGG colonization resulted in changing the bacterial composition in 3-week-old mice to a similar abundance pattern in 8-week-old non-colonized and colonized mice.

Thus, the results that LGG colonization in mice elicited a shift of the diversity, richness, and the composition of the intestinal microbiota at early stage of life to an adult-like microbiota suggest that LGG colonization facilitates maturation of the intestinal microbiota, which may exert beneficial effects on intestinal development.

\section{LGG colonization prevents dextran sodium sulfate-} induced intestinal injury and inflammation in adult mice

It has been reported that immune effects of microbial exposure at early life are persist into later stage of life and associated with prevention of inflammatory bowel disease in humans ${ }^{40}$ and animals. ${ }^{41}$ Therefore, we asked whether neonatal LGG colonization prevented intestinal inflammation in adult mice. Eight-week-old mice were treated with 3\% dextran sodium sulfate (DSS) in drinking water for 4 days. DSS treatment induced colonic injury and acute colitis with massive ulceration, crypt damage, and severe inflammation in non-colonized mice (score: $7.68 \pm 1.5$ ). These abnormalities were significantly reduced in mice with LGG colonization (score: $3.81 \pm 1.1$, $P<0.05$ ) (Figure 6a,b). DSS-induced shortening of the colon, a feature of intestinal inflammation, in non-colonized mice was prevented in LGG-colonized mice (Figure 6c).

To further understand the effects of LGG colonization on colitis, markers of colitis in this model were examined. Increased proinflammatory cytokine production, such as 
a

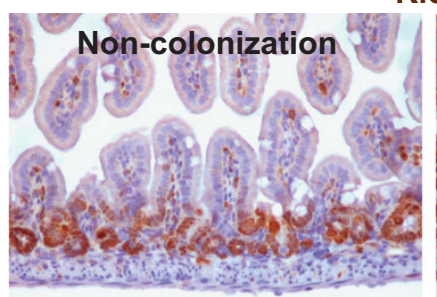

Ki67

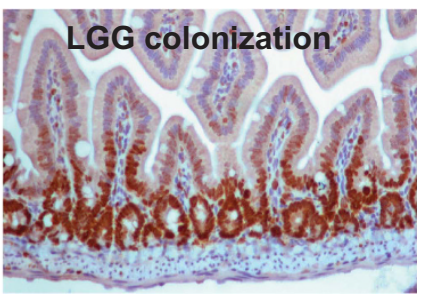

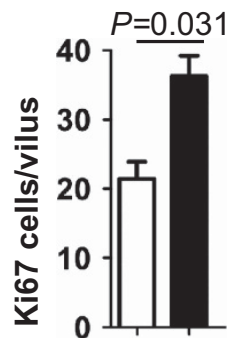

b

MUC2
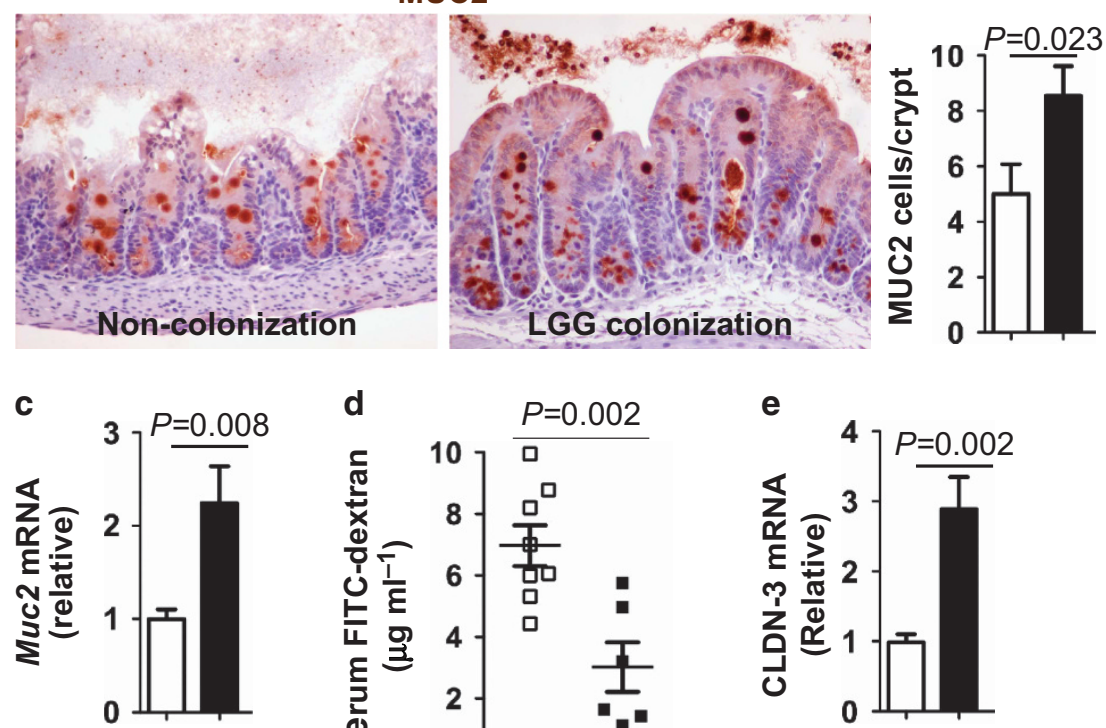

d

d

$P=0.002$

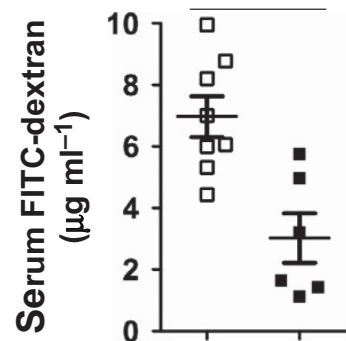

e

$4] P=0.002$

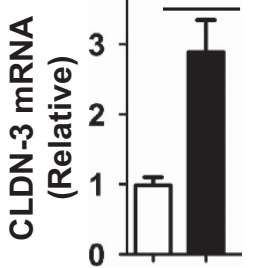

f

ZO-1/DAPI
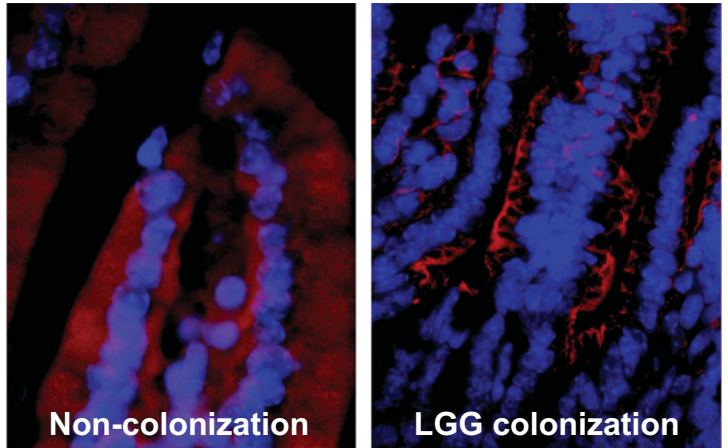

Figure 3 Lactobacillus rhamnosus GG (LGG) colonization enhances proliferation, differentiation, and tight junction formation in 2-week-old mice. Mice were treated as described in Figure 2. Proliferation (Ki67, a) in the small intestine and differentiation (MUC2, b) in the colon were assessed using immunostaining. The numbers of positively stained cells were shown. Real-time PCR analysis of Muc2 expression in colonic epithelial cells was shown (c). Barrier formation was detected by the in vivo FITC-dextran assay. The FITC-dextran level in serum is shown (d). Real-time PCR analysis was performed to detect CLND-3 expression in the small intestinal epithelial cells (e). Paraffin-embedded small intestinal tissues were used to determine ZO-1 distribution by immunohistochemistry using an anti-ZO-1 antibody and FITC-labeled secondary antibody and visualized by fluorescence microcopy (red staining). Nuclei were stained with DAPI (blue staining) (f). In (c) and (e), the average of mRNA expression levels in the non-colonized mice was set as $100 \%$, and the mRNA expression level of each mouse was compared with this average. In (a-c) and (e), $n=7$ in each group. Images in (f) represent five mice in each group.

TNF, KC, and IL-6, is a hallmark of DSS-induced colitis. ${ }^{42,43}$ Therefore, we tested the effects of LGG colonization on inflammatory cytokine production in the DSS-induced colitis model. We used real-time PCR analysis of RNA isolated from the colon to detect proinflammatory cytokine mRNA levels.
TNF, KC, and IL-6 mRNA levels were significantly increased in non-colonized mice, which were reduced in LGG-colonized mice (Figure 6d). Disruption of intestinal integrity is a character of DSS-induced intestinal injury and colitis. DSS induced increased permeability, as determined by the level of 
a

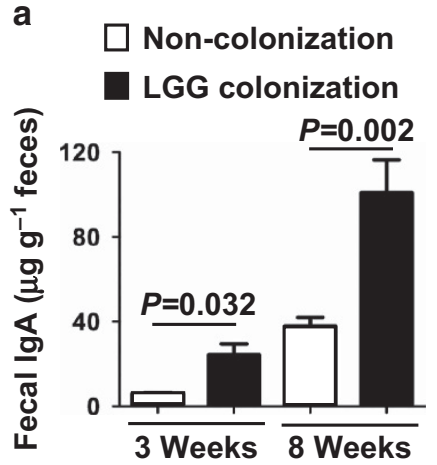

b

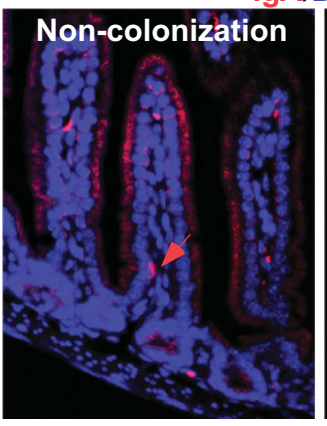

IgA/DAPI

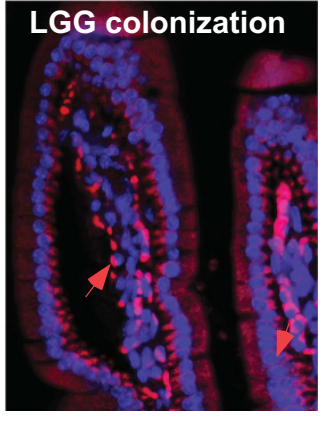

LGG colonization$$
\text { C }
$$

Non-colonization

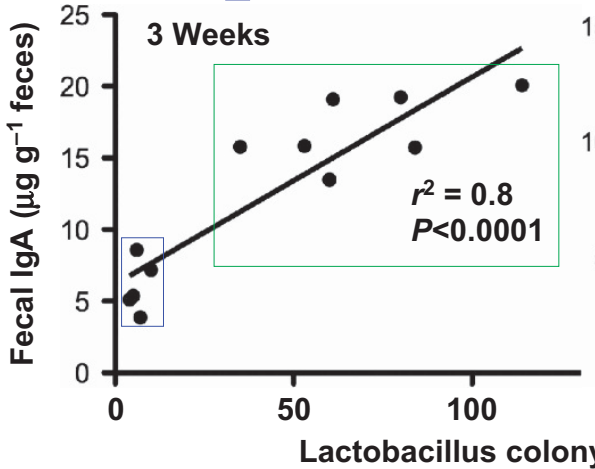

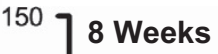

Figure 4 Lactobacillus rhamnosus GG(LGG) colonization increases production of IgA in the intestine. Mice were treated as described in Figure 2, and feces were collected at the indicated ages. Fecal slgA levels were determined by ELISA. $N=10$ in non-colonization group, $N=14$ in LGG-colonized group (a). The small intestinal tissues from 3-week-old pups were prepared for IgA immunostaining (b). Red arrows indicate IgA positively stained lamina propria cells. Images in (b) represent five mice in each group. Feces were collected from mice with indicated ages and cultured on deMan, Rogosa and Sharpe agar to determine the number of Lactobacillus colonies. The number of fecal Lactobacillus colonies was plotted against the fecal slgA level for each mouse. The lines illustrate the best-fit linear regressions with the correlation coefficient and significance as indicated (c).

FITC-dextran in the serum, in non-LGG colonized mice, which was attenuated in mice with LGG colonization (Figure 6e).

More importantly, the number of Lactobacillus in feces was negatively correlated with the inflammation/injury score in mice with LGG colonization (Figure 6f). These results indicate that the effects of LGG colonization on intestinal development, maturation of the microbiota, and IgA production are associated with long-term health outcomes for protection against intestinal inflammation.

\section{DISCUSSION}

One important factor that affects the actions of probiotics in vivo is the bioavailability. In most animal studies, mice are given LGG via daily gavage. However, the viability of probiotics in the intestinal tract delivered through the oral route is a concern for assessing probiotic clinical efficacy. Therefore, approaches that assure survival of probiotics in the gastrointestinal tract could increase the clinical efficacy of probiotics. Thus, our study was focused on developing a model of LGG colonization in conventional mice and investigating its effects on intestinal development and the potential lifelong health consequences.

The microbial environment at birth, which is shaped by the mode of delivery, significantly affects colonization. ${ }^{2}$ This evidence suggests that there is a narrow window of opportunity shortly after birth for colonization. In fact, studies have shown that LGG persisted in the stools only for 7-14 days after discontinuing feeding infants with formula supplemented with LGG for 7 days. ${ }^{44}$ In our study, by introduction of LGG to mice from postnatal days 1 to 5 , we developed a mouse model of LGG colonization in conventionally raised mice, which could persist in 8-week-old mice. These findings suggest that postnatal days $1-5$ is a proper time frame for bacterial colonization in conventionally raised mice. Currently, most reported studies were focused on colonization of germ-free animals with bacteria. However, human and animals are naturally colonized by commensal bacteria after birth and it is questionable whether studies using germ-free animals can be extrapolated to the physiological condition. Thus, successful colonization of conventional mice with LGG provides a useful strategy to study the role of commensal bacteria and probiotics in regulation of intestinal development and protective immunity under physiological conditions.

Currently, studies regarding host factors that contribute to bacterial colonization are limited. Polysaccharide A from Bacteroides fragilis has been reported to activate the TLR pathway in $\mathrm{CD} 4^{+} \mathrm{T}$ cells to promote immunologic tolerance for $B$. fragilis colonization in germ-free mice. ${ }^{45}$ As we have 

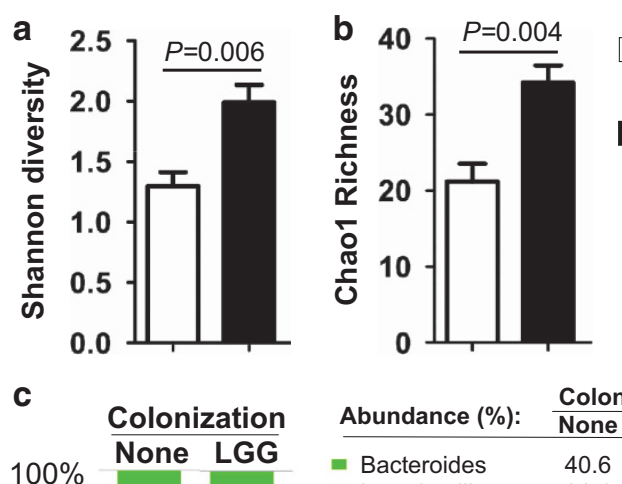

\begin{tabular}{|c|c|c|c|}
\hline \multirow{2}{*}{ Abundance (\%): } & \multicolumn{2}{|c|}{ Colonization } & \multirow{2}{*}{$P$ value } \\
\hline & None & LGG & \\
\hline - Bacteroides & 40.6 & 7.7 & $<0.001$ \\
\hline - Lactobacillus & 31.4 & 42.6 & 0.031 \\
\hline = Clostridium & 15.2 & 29.3 & 0.017 \\
\hline Helicobacter & 8.9 & 0.5 & $<0.001$ \\
\hline $\begin{array}{l}\text { Enterobacteriacae } \\
\text { unknown }\end{array}$ & 1.2 & $<0.1$ & 0.028 \\
\hline Parabacteroides & 1.1 & 5.6 & 0.187 \\
\hline = No hit & 0.8 & 1.4 & 0.374 \\
\hline Parasutteralla & 0.7 & 0.1 & $<0.001$ \\
\hline $\begin{array}{l}\text { Clostridials } \\
\text { unknown }\end{array}$ & 0 & 2.7 & $<0.001$ \\
\hline Roseburia & 0 & 1.9 & 0.044 \\
\hline - Anaerotruncus & 0 & 1.6 & 0.004 \\
\hline Ureaplasma & 0 & 1.4 & 0.063 \\
\hline - Oscillospira & 0 & 1.1 & 0.037 \\
\hline $\begin{array}{l}\text { Bacteroidales } \\
\text { unknown }\end{array}$ & 0 & 1.1 & $<0.001$ \\
\hline = Mucispirilium & 0 & 0.9 & 0.069 \\
\hline Prevotella & 0 & 0.8 & 0.063 \\
\hline $\begin{array}{l}\text { Hydrogeno } \\
\text { anaerobacterium }\end{array}$ & 0 & 0.4 & 0.096 \\
\hline = Bryantella & 0 & 0.3 & 0.044 \\
\hline $\begin{array}{l}\text { Candidatus } \\
\text { Arthromitus }\end{array}$ & 0 & 0.1 & 0.242 \\
\hline = Oscillibacter & 0 & 0.1 & 0.002 \\
\hline - Alistipes & 0 & 0.1 & 0.347 \\
\hline
\end{tabular}

Figure 5 Lactobacillus rhamnosus GG (LGG) colonization modulates LGG colonization benefits maturation of the intestinal microbiota in 3-week-old mice. Mice were treated as described in Figure 2. Genomic DNA was extracted from colonic tissues of 3-week-old pups to assess the community composition of the mucosally-adherent microbiota by 454based 16S rRNA amplicon library sequencing. Shannon diversity (a) and Chao1 richness (b), and detailed relative abundance of bacterial taxa at the genus level (c) are shown. $n=5$ in each group.

found that LGG-derived p40 activates epidermal growth factor receptor activation in intestinal epithelial cells, ${ }^{27,46}$ we conducted experiments to colonize LGG in Egfrwa5 mice with defective epidermal growth factor receptor kinase. However, none of Egfr $r^{\text {wa5 }}$ mice $(n=7)$ could be colonized with LGG. This evidence suggests that epidermal growth factor receptor signaling may have a role in LGG colonization. We have reported that activation of epidermal growth factor receptor by p40 stimulated mucin production ${ }^{26}$ and increased Muc2 expressing cells in LGG-colonized mice were found in this study. Mucin has been shown to mediate bacterial growth. Both commensal and pathogenic bacteria benefit from their ability to regulate mucin synthesis and/or secretion in host goblet cells. ${ }^{47,48}$ Thus, LGG-stimulated mucin production may contribute to LGG colonization.

The process for developing initial microbiota community is driven by complex interactions, including the mother's microbiota, mode of delivery, type of feeding, the environment, and use of antibiotics and disease. ${ }^{49}$ In addition to oligosaccharides in breast milk, which have been shown to support the establishment of a healthy microbiota in breast-fed infants, ${ }^{50,51}$ mother-derived secretory IgA in breast milk has been reported to shape the composition of the intestinal microbiota in neonatal mice at the age of weaning, as compared with that in neonates that did not receive maternal secretory IgA. These differences were magnified when the mice reached adulthood. ${ }^{52}$ Furthermore, a study using different genetic background mice showed that $\mathrm{BALB} / \mathrm{c}$ mice had significant difference in abundance of bacterial species and higher diversity as compared with $\mathrm{C} 57 \mathrm{BL} / 6$ mice, which was correlated with higher abundance and diversity of IgAs in BALB/c mice than in C57BL/6 mice. ${ }^{53}$ This study also demonstrated polyreactive IgA-mediated entrance of non-invasive bacteria to Peyer's patches contributed to the induction of bacteria-specific IgA and the establishment of a positive feedback loop of IgA production. ${ }^{53}$ Thus, upregulation of IgA production by LGG colonization may have roles in increasing diversity and richness of the microbiota in neonatal mice. However, our results showed that LGG colonization elicited changes in bacterial composition, but did not affect the diversity and richness of the microbiota in adult mice. Similar results were reported in a human study that perinatal supplementation of LGG to mothers had no major impacts on later microbiota development in children. ${ }^{54}$ Development of the intestinal microbiota is a dynamic process, and the microbiota diversity in adults is genetically and environmentally driven. ${ }^{55}$ LGG colonizationinduced effects may not serve as a major factor for determining microbiota diversity in adult mice, although increased $\operatorname{IgA}$ production may mediate promoting maturation of the intestinal microbiota in neonatal mice, as shown in our study. In addition to IgA production, LGG colonization may exert other functions that contribute to regulation of the intestinal microbiota at the early stage and in adulthood, which is worthy of further investigation.

Results from this study suggest that LGG colonization led to several outcomes that may contribute to maintaining health and disease prevention. LGG colonization increased the abundance of Lactobacillus and Clostridium in 3-week-old mice. Studies have demonstrated that Faecalibacterium prausnitzii, an intestinal bacterium belonging to the Clostridium cluster, was reduced in abundance in Crohn's disease, ${ }^{56}$ and Clostridium butyricum, as a probiotic, induces interleukin-10 production in macrophages in inflamed mucosa and prevents acute experimental colitis. ${ }^{57}$ This evidence supports the contribution of LGG colonization to host health through regulation of the intestinal microbiota.

Our results demonstrate that the effects of LGG colonization on increased IgA production can persist in adult mice. Mechanisms underlying IgA in breast milk promotes lifelong intestinal homeostasis have been demonstrated in mice. Early exposure to maternal secretory IgA prevented the translocation of aerobic bacteria from the neonatal gut into draining lymph nodes and resulted in ameliorating DSSinduced colonic damage in adult mice, which was associated 

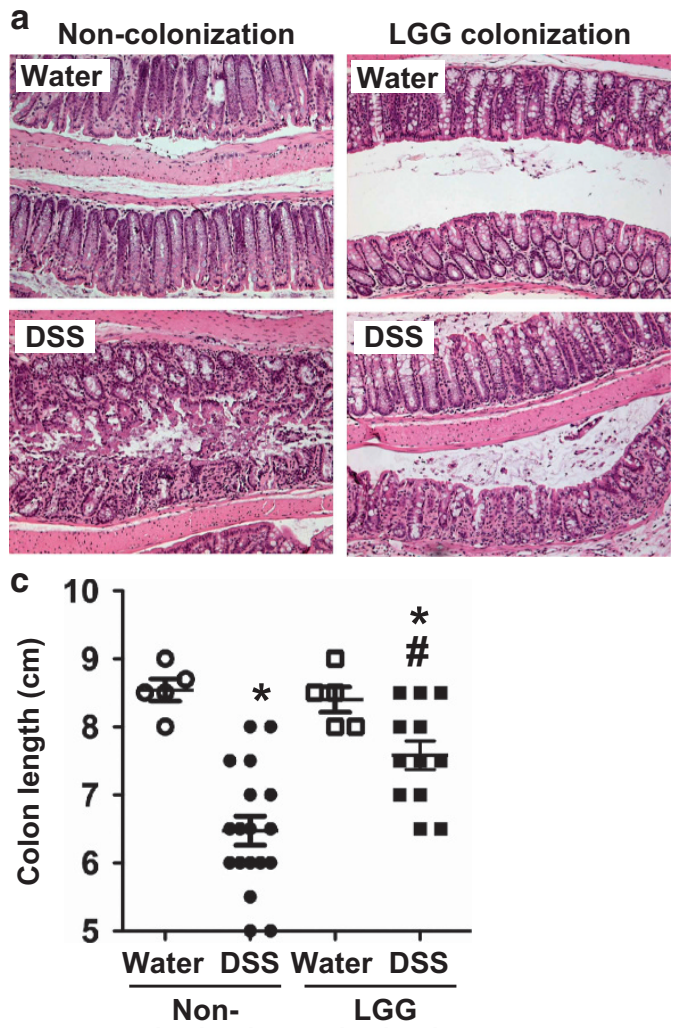

colonization colonization
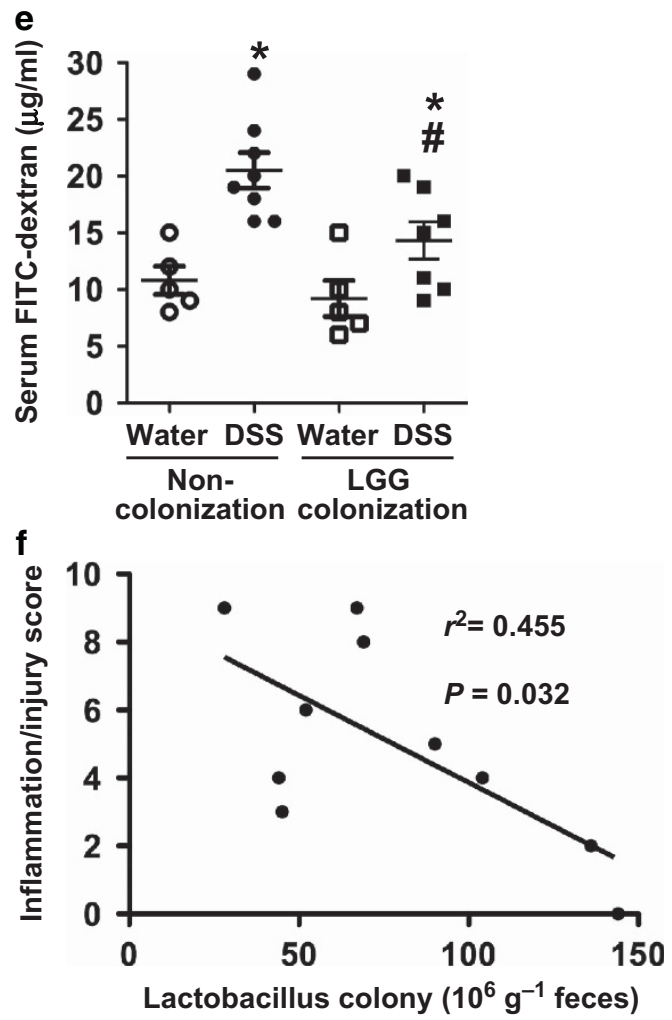
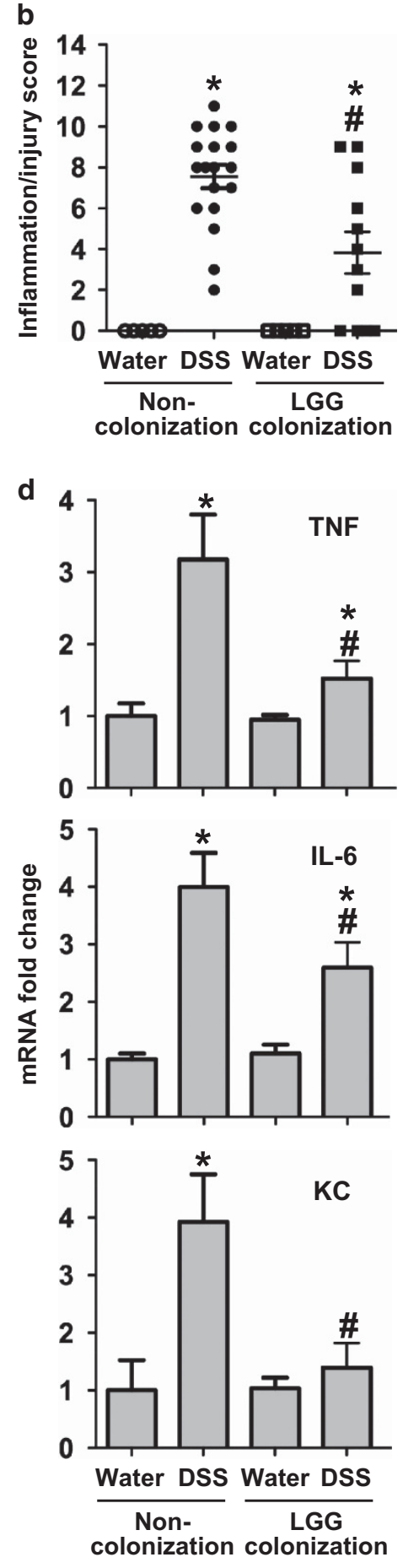
with upregulation of expression of genes involved in cell metabolism and repair intestinal epithelial cells. ${ }^{52}$ Thus, this evidence supports the possibility that increased IgA production by LGG colonization may contribute to decreasing susceptibility to colitis in adults.

It is important to study the mechanisms by which LGG colonization increases IgA production. APRIL(A proliferationinducing ligand), a tumor necrosis factor family member, has a role in directing B-cell class switching toward IgA secreting plasma cells. ${ }^{58,59}$ Our preliminary studies have shown that $\mathrm{p} 40$, an LGG-derived protein, ${ }^{26-28}$ upregulated APRIL expression in intestinal epithelial cells, which contributed to promoting IgA production by B cells (unpublished data). This mechanism may be involved in LGG colonization-enhanced IgA production because APRIL expression in small intestinal epithelial cells was upregulated in LGG-colonized mice (Supplementary Figure 3).

Results from this study demonstrated that some effects of LGG colonization are limited to the early postnatal period, including accelerating bodyweight gain, and intestinal epithelial cell proliferation and differentiation. In fact, a clinical study demonstrated that perinatal LGG supplementation to mothers did not affect the weight gain in children remaining normal bodyweight over the 10-year follow-up. However, in children who became overweight by the age of 10 years, there was a tendency that the body mass index of children whose mothers received LGG were constantly lower until 7 years of age as compared with children whose mothers received placebo. ${ }^{60}$ Therefore, LGG colonization is not likely to result in overweight and may have beneficial effects on prevention of obesity in the later life.

In summary, these findings elucidate the impact of neonatal colonization of mice with LGG on intestinal development and long-term consequence in the adult as protection against colitis. The approach of LGG colonization applied in this study should be useful for delivery of other probiotics to increase their bioavailability and beneficial effects on the host.

\section{METHODS}

Mice and LGG colonization. LGG (American Type Culture Collection 53103) was cultured in Lactobacillus MRS broth. Fixation of LGG was performed using $0.25 \%$ glutaraldehyde in PBS for $15 \mathrm{~min}$ at room temperature followed by two washings with $0.1 \mathrm{M}$ glycine to inactive free aldehyde and three washings with PBS. ${ }^{61}$
Paired pregnant C57BL/6 female mice from the same litter were housed in the same cage until gestational day 18 . Then pregnant mice were separated into different cages and gavaged with $10^{8} \mathrm{cfu}$ live LGG (Live-LGG) or glutaraldehyde fixed-LGG (Fix-LGG) until delivery. Pups were fed with $10^{7}$ cfu Live-LGG or Fix-LGG for 5 days starting at postnatal days 1,3 , and 5 . All animal experiments were performed according to protocols approved by the Institutional Animal Care and Use Committee at Vanderbilt University.

Bodyweight was recorded at birth and every week. Percentage of change compared with the bodyweight at birth was used to evaluate the growth rate. Intestinal tissues from 2- and 3-week-old mice were isolated for preparation of paraffin-embedded tissue sections for immunostaining and hematoxylin and eosin (H\&E) staining. Villus length and crypt depth were measured using H\&E-stained sections.

To detect fecal lactobacillus, mouse feces were solubilized in MRS broth and supernatants were cultured on MRS agar. The number of fecal Lactobacillus colony was counted.

Determining LGG colonization by DNA fingerprint analysis. To detect LGG in fecal bacteria, feces were cultured in MRS broth. Genomic DNA was isolated from cultured bacteria using the Wizard Genomic DNA Purification Kit (Promega Corporation, Madison, WI). DNA was used as a template for PCR amplification of 16S rRNA gene using L. rhamnosus-specific primers (CAGACTGAAAGTCTGACGG and GCGATGCGAATTTCTATTATT). ${ }^{30,31}$ PCR products were separated using denaturing gradient gel electrophoresis to determine their migration profiles. Genomic DNA from LGG was used as a control. Nucleotide sequences of PCR products were further confirmed by direct sequencing using primers for PCR amplification.

LGG colonization was defined as detection of LGG in fecal bacteria by DNA fingerprint analysis. The colonization rate of individual litter was determined by the percentage of mice with LGG colonization in the total number of mice from the same litter.

Fecal IgA detection by ELISA. Fecal homogenates were prepared by solubilizing feces in PBS (10\% wt:vol) and saved at $-80^{\circ} \mathrm{C}$. 96-well plates were coated with an anti-mouse IgA antibody (Sigma-Aldrich, St. Louis, MO) in PBS at $4{ }^{\circ} \mathrm{C}$ overnight. Non-specific protein binding was blocked by incubating plates with $1 \%$ of bovine serum albumin in PBS for $1 \mathrm{~h}$ at room temperature. Plates were then incubated with supernatants of the fecal homogenates for $1 \mathrm{~h}$, followed by an FITCconjugated anti-mouse IgA antibody (Sigma-Aldrich) for $1 \mathrm{~h}$ at room temperature. Fluorescent intensity was measured using a fluorescent plate reader. Purified mouse IgA (Sigma-Aldrich) was used for generating a standard calibration curve.

Intestinal permeability assay. To detect permeability in 2-week-old mice, the abdomen was opened and a ligation was performed in the ileum at $2 \mathrm{~mm}$ above the cecum. FITC-conjugated dextran dissolved in water $(4,000 \mathrm{~mol} \mathrm{wt}$, Sigma-Aldrich) was injected into the ileum at $4 \mathrm{mg}$ per $10 \mathrm{~g}$ bodyweight. Then, the abdomen was closed. In adult mice with DSS treatment, colitis occurred in the colon. A ligation was performed in the colon at $2 \mathrm{~mm}$ below the cecum, then FITC-dextran was injected into the colon. Whole blood was collected using

Figure 6 Colonization with Lactobacillus rhamnosus GG (LGG) prevents colitis in adult conventionally raised mice. Mice were treated as described in Figure 2. Eight-week-old mice were treated with $3 \%$ dextran sodium sulfate (DSS) in drinking water for 4 days. Paraffin-embedded colon sections were stained with $\mathrm{H} \& \mathrm{E}$ for light microscopic assessment of epithelial damage and inflammation (a). The inflammation/injury scores are shown (b). The length of colon was measured (c). mRNA was isolated from the colonic tissues for real-time PCR analysis of indicated cytokine mRNA expression levels. The average of cytokine mRNA expression levels in the water group of non-colonized mice was set as $100 \%$, and the mRNA expression level of each mouse was compared with this average (d). Intestinal permeability was determined by an in vivo permeability assay on the fourth day of DSS treatment. FITCdextran level in sera is shown (e). ${ }^{*} P<0.01$ compared with the water-treated mice in the non-colonization group. ${ }^{\#} P<0.05$ compared with the noncolonized mice with DSS treatment. Feces were collected from LGG-colonized mice before DSS treatment and cultured on deMan, Rogosa and Sharpe agar to determine the number of Lactobacillus colonies. The number of fecal Lactobacillus colony was plotted against the inflammation and injury score for each mouse. The lines illustrate the best-fit linear regressions with the correlation coefficient and significance as indicated (f). 
heparinized micro-hematocrit capillary tubes via eye bleed $2 \mathrm{~h}$ after FITC-dextran administration. Fluorescence intensity in sera was analyzed using a plate reader. The concentration of FITC-dextran in sera was determined by comparing with the FITC-dextran standard curve.

Microbiota analysis. Frozen colonic tissues from 3-week-old pups and cecum feces from 8-week-old mice were sent to the Research and Testing Laboratory, LLC (Lubbock, TX) for metagenomics data collection. The 16S RNA samples were extracted on site and subjected to tag-encoded FLX amplicon pyrosequencing at a $3-\mathrm{K}$ average coverage. Raw metagenomics data were analyzed using Qiime ${ }^{62}$ (http://www.qiime.org) including 454-based 16S rRNA amplicon library sequencing. Diversity was examined from two perspectives, overall richness, which was expressed as the number of operational taxonomic units and quantified using the Chaol richness estimator, and overall diversity, which was expressed as Shannon Diversity. Measures of diversity were screened for group differences by an analysis of variance (ANOVA). Individual operational taxonomic units were examined for significant changes between non-colonized and colonized groups. Operational taxonomic unit count data were analyzed using a generalized linear model with a negative binomial distribution. Multivariate differences among groups were evaluated with "Permutational Multivariate Analysis of Variance Using Distance Matrices," function adonis. For ADONIS, distances among samples first were calculated using unweighted or weighted UniFrac, and then an ANOVA-like simulation was conducted to test for group differences.

Intestinal epithelial cell isolation. Small intestinal and colonic epithelial cells were isolated as described before. ${ }^{27}$ Detailed experimental procedures are provided in the Supplementary Material

Real-time PCR assay. Total RNA was isolated from small intestinal, colonic epithelial cells, and colonic tissues for real-time PCR analysis of MUC2, CLDN3, TNF, IL-6, and KC gene expression. Detailed experimental procedures are provided in the Supplementary Material.

Immunohistochemistry. Paraffin-embedded tissue sections were used for immunohistochemistry to detect cells expression of Ki67, MUC2, and IgA, and ZO-1 localization. Detailed experimental procedures are provided in the Supplementary Material.

Analysis of intestinal injury and colitis. Eight-week-old mice were administered 3\% DSS (molecular weight 36-50 kDa, MP Biomedicals, Santa Ana, CA) in drinking water for 4 days. Mice were fed with drinking water as a control for DSS treatment. Paraffin-embedded colon tissue sections were stained with hematoxylin and eosin for light microscopic examination to assess colon injury and inflammation. Samples from the entire colon were examined by a pathologist blinded to treatment conditions. A modified combined scoring system ${ }^{63}$ including degree of inflammation (scale of $0-3$ ) and crypt damage $(0-4)$, percentage of area involved by inflammation (0-4) and crypt damage (0-4), and depth of inflammation (0-3) was applied for assessing intestinal injury and colitis.

Statistical analysis. Statistical significance was determined by oneway ANOVA followed by Newman-Keuls analysis using Prism 5.0 (GraphPad Software, San Diego, CA) for multiple comparisons and $T$-test for paired samples. A $P$-value of $<0.05$ was defined as statistically significant. All data are presented as mean \pm s.e.m.

SUPPLEMENTARY MATERIAL is linked to the online version of the paper at http://www.nature.com/mi

\section{ACKNOWLEDGMENTS}

This work was supported by National Institutes of Health $(\mathrm{NIH})$ grants R01DK081134 (F.Y.), R01DK56008 and R01DK54993 (D.B.P.), National
Nature Science Foundation of China grants 81470796 (F.Y.) and 81300272 (H.C.), Tianjin Research Program of Application Foundation and Advanced Technology of China grant 15JCZDJC36600 (F.Y.), and core services performed through Vanderbilt University Medical Center's Digestive Disease Research Center supported by NIH grant P30DK058404.

\section{DISCLOSURE}

The authors declared no conflict of interest.

c) 2017 Society for Mucosal Immunology

\section{REFERENCES}

1. Aagaard, K., Ma, J., Antony, K.M., Ganu, R., Petrosino, J. \& Versalovic, J. The placenta harbors a unique microbiome. Sci. Transl. Med. 6, 237ra265 (2014).

2. Dominguez-Bello, M.G. et al. Delivery mode shapes the acquisition and structure of the initial microbiota across multiple body habitats in newborns. Proc. Natl. Acad. Sci. USA 107, 11971-11975 (2010).

3. Nicholson, J.K. et al. Host-gut microbiota metabolic interactions. Science 336, 1262-1267 (2012).

4. Walker, W.A. Initial intestinal colonization in the human infant and immune homeostasis. Ann. Nutr. Metab. 63, 8-15 (2013).

5. Haberman, Y. et al. Pediatric Crohn disease patients exhibit specific ileal transcriptome and microbiome signature. J. Clin. Invest. 124, 3617-3633 (2014).

6. Hansen, R. et al. Microbiota of de-novo pediatric IBD: increased Faecalibacterium prausnitzii and reduced bacterial diversity in Crohn's but not in ulcerative colitis. Am. J. Gastroenterol. 107, 1913-1922 (2012).

7. Michail, S. et al. Alterations in the gut microbiome of children with severe ulcerative colitis. Inflamm. Bowel. Dis. 18, 1799-1808 (2012).

8. Raymond, F. et al. The initial state of the human gut microbiome determines its reshaping by antibiotics. ISME J. 10, 707-720 (2016).

9. Kronman, M.P., Zaoutis, T.E., Haynes, K., Feng, R. \& Coffin, S.E. Antibiotic exposure and IBD development among children: a population-based cohort study. Pediatrics 130, e794-e803 (2012).

10. Berg, R.D. The indigenous gastrointestinal microflora. Trends Microbiol. 4, 430-435 (1996).

11. Hooper, L.V., Wong, M.H., Thelin, A., Hansson, L., Falk, P.G. \& Gordon, J.I. Molecular analysis of commensal host-microbial relationships in the intestine. Science 291, 881-884 (2001).

12. Cox, L.M. et al. Altering the intestinal microbiota during a critical developmental window has lasting metabolic consequences. Cell 158, 705-721 (2014).

13. Preidis, G.A., Hill, C., Guerrant, R.L., Ramakrishna, B.S., Tannock, G.W. \& Versalovic, J. Probiotics, enteric and diarrheal diseases, and global health. Gastroenterology 140, 8-14 (2011).

14. Guandalini, S. Probiotics for prevention and treatment of diarrhea. J. Clin. Gastroenterol. 45, S149-S153 (2011).

15. Sanders, M.E. et al. An update on the use and investigation of probiotics in health and disease. Gut 62, 787-796 (2013).

16. Patel, R.M., Myers, L.S., Kurundkar, A.R., Maheshwari, A., Nusrat, A. \& Lin, P.W. Probiotic bacteria induce maturation of intestinal claudin 3 expression and barrier function. Am. J. Pathol. 180, 626-635 (2012).

17. Preidis, G.A. et al. Probiotics stimulate enterocyte migration and microbial diversity in the neonatal mouse intestine. FASEB J. 26, 1960-1969 (2012)

18. Becker, S. et al. Bacteria regulate intestinal epithelial cell differentiation factors both in vitro and in vivo. PLOS One 8, e55620 (2013).

19. Forestier, C., De Champs, C., Vatoux, C. \& Joly, B. Probiotic activities of Lactobacillus casei rhamnosus: in vitro adherence to intestinal cells and antimicrobial properties. Res. Microbiol. 152, 167-173 (2001).

20. Dotterud, C.K. et al. Does maternal perinatal probiotic supplementation alter the intestinal microbiota of mother and child? J. Pediatr. Gastroenterol. Nutr. 61, 200-207 (2015).

21. Kalliomaki, M., Salminen, S., Arvilommi, H., Kero, P., Koskinen, P. \& Isolauri, E. Probiotics in primary prevention of atopic disease: a randomised placebo-controlled trial. Lancet 357, 1076-1079 (2001). 
22. Kalliomaki, M., Salminen, S., Poussa, T., Arvilommi, H. \& Isolauri, E. Probiotics and prevention of atopic disease: 4-year follow-up of a randomised placebo-controlled trial. Lancet 361, 1869-1871 (2003).

23. Kalliomaki, M., Salminen, S., Poussa, T. \& Isolauri, E. Probiotics during the first 7 years of life: a cumulative risk reduction of eczema in a randomized, placebo-controlled trial. J. Allergy Clin. Immunol. 119, 1019-1021 (2007).

24. Scalabrin, D.M., Johnston, W.H., Hoffman, D.R., P'Pool, V.L., Harris, C.L. \& Mitmesser, S.H. Growth and tolerance of healthy term infants receiving hydrolyzed infant formulas supplemented with Lactobacillus rhamnosus GG: randomized, double-blind, controlled trial. Clin. Pediatr. 48, 734-744 (2009).

25. Doron, S., Snydman, D.R. \& Gorbach, S.L. Lactobacillus GG: bacteriology and clinical applications. Gastroenterol. Clin. North Am. 34, 483-498 (2005).

26. Wang, L. et al. Activation of epidermal growth factor receptor mediates mucin production stimulated by $\mathrm{p} 40$, a Lactobacillus rhamnosus GGderived protein. J. Biol. Chem. 289, 20234-20244 (2014).

27. Yan, F. et al. Colon-specific delivery of a probiotic-derived soluble protein ameliorates intestinal inflammation in mice through an EGFR-dependent mechanism. J. Clin. Invest. 121, 2242-2253 (2011).

28. Yan, F., Cao, H., Cover, T.L., Whitehead, R., Washington, M.K. \& Polk, D.B. Soluble proteins produced by probiotic bacteria regulate intestinal epithelial cell survival and growth. Gastroenterology 132, 562-575 (2007).

29. Hansen, C.H. et al. Patterns of early gut colonization shape future immune responses of the host. PLoS One 7, e34043 (2012).

30. Guan, L.L., Hagen, K.E., Tannock, G.W., Korver, D.R., Fasenko, G.M. \& Allison, G.E. Detection and identification of Lactobacillus species in crops of broilers of different ages by using PCR-denaturing gradient gel electrophoresis and amplified ribosomal DNA restriction analysis. Appl. Environ. Microbiol. 69, 6750-6757 (2003).

31. Walter, J., Heng, N.C., Hammes, W.P., Loach, D.M., Tannock, G.W. \& Hertel, C. Identification of Lactobacillus reuteri genes specifically induced in the mouse gastrointestinal tract. Appl. Environ. Microbiol. 69, 20442051 (2003).

32. Ahlroos, T. \& Tynkkynen, S. Quantitative strain-specific detection of Lactobacillus rhamnosus GG in human faecal samples by real-time PCR. J. Appl. Microbiol. 106, 506-514 (2009).

33. Agarwal, R. et al. Effects of oral Lactobacillus GG on enteric microflora in low-birth-weight neonates. J. Pediatr. Gastroenterol. Nutr. 36, 397-402 (2003).

34. Seth, A., Yan, F., Polk, D.B. \& Rao, R.K. Probiotics ameliorate the hydrogen peroxide-induced epithelial barrier disruption by a PKC- and MAP kinasedependent mechanism. Am. J. Physiol. Gastrointest. Liver Physiol. 294, G1060-G1069 (2008).

35. Yoda, K., Miyazawa, K., Hosoda, M., Hiramatsu, M., Yan, F. \& He, F. Lactobacillus GG-fermented milk prevents DSS-induced colitis and regulates intestinal epithelial homeostasis through activation of epidermal growth factor receptor. Eur. J. Nutr. 53, 105-115 (2014).

36. Pabst, O. New concepts in the generation and functions of IgA. Nat. Rev. Immunol. 12, 821-832 (2012).

37. Chattha, K.S. et al. Probiotics and colostrum/milk differentially affect neonatal humoral immune responses to oral rotavirus vaccine. Vaccine $\mathbf{3 1}$, 1916-1923 (2013).

38. de Vrese, M., Rautenberg, P., Laue, C., Koopmans, M., Herremans, T. \& Schrezenmeir, J. Probiotic bacteria stimulate virus-specific neutralizing antibodies following a booster polio vaccination. Eur. J. Nutr. 44, 406-413 (2005).

39. Yatsunenko, T. et al. Human gut microbiome viewed across age and geography. Nature 486, 222-227 (2012).

40. Lopez-Serrano, P., Perez-Calle, J.L., Perez-Fernandez, M.T., FernandezFont, J.M., Boixeda de Miguel, D. \& Fernandez-Rodriguez, C.M. Environmental risk factors in inflammatory bowel diseases. Investigating the hygiene hypothesis: a Spanish case-control study. Scand. J. Gastroenterol. 45, 1464-1471 (2010).

41. Olszak, T. et al. Microbial exposure during early life has persistent effects on natural killer T cell function. Science 336, 489-493 (2012).
42. Egger, B., Bajaj-Elliott, M., MacDonald, T.T., Inglin, R., Eysselein, V.E. \& Buchler, M.W. Characterisation of acute murine dextran sodium sulphate colitis: cytokine profile and dose dependency. Digestion 62, 240-248 (2000).

43. Garside, P. Cytokines in experimental colitis. Clin. Exp. Immunol. 118, 337-339 (1999).

44. Petschow, B.W., Figueroa, R., Harris, C.L., Beck, L.B., Ziegler, E. \& Goldin, B. Effects of feeding an infant formula containing Lactobacillus GG on the colonization of the intestine: a dose-response study in healthy infants. J. Clin. Gastroenterol. 39, 786-790 (2005).

45. Round, J.L. et al. The Toll-like receptor 2 pathway establishes colonization by a commensal of the human microbiota. Science 332, 974-977 (2011).

46. Yan, F. et al. A Lactobacillus rhamnosus GG-derived soluble protein, p40, stimulates ligand release from intestinal epithelial cells to transactivate epidermal growth factor receptor. J. Biol. Chem. 288, 30742-30751 (2013).

47. Dharmani, P., Srivastava, V., Kissoon-Singh, V. \& Chadee, K. Role of intestinal mucins in innate host defense mechanisms against pathogens. J. Innate Immun. 1, 123-135 (2009).

48. Martens, E.C., Roth, R., Heuser, J.E. \& Gordon, J.I. Coordinate regulation of glycan degradation and polysaccharide capsule biosynthesis by a prominent human gut symbiont. J. Biol. Chem. 284, 18445-18457 (2009).

49. Backhed, F. et al. Dynamics and stabilization of the human gut microbiome during the first year of life. Cell Host Microbe 17, 690-703 (2015).

50. Martin, R. et al. Cultivation-independent assessment of the bacterial diversity of breast milk among healthy women. Res. Microbiol. 158, 31-37 (2007).

51. Martin, R. et al. Isolation of bifidobacteria from breast milk and assessment of the bifidobacterial population by PCR-denaturing gradient gel electrophoresis and quantitative real-time PCR. Appl. Environ. Microbiol. 75, 965969 (2009).

52. Rogier, E.W. et al. Secretory antibodies in breast milk promote long-term intestinal homeostasis by regulating the gut microbiota and host gene expression. Proc. Natl. Acad. Sci. USA 111, 3074-3079 (2014).

53. Fransen, F. et al. BALB/c and C57BL/6 mice differ in polyreactive IgA abundance, which impacts the generation of antigen-specific IgA and microbiota diversity. Immunity 43, 527-540 (2015).

54. Endo, A., Prtty, A., Kalliomki, M., Isolauri, E. \& Salminen, S. Long-term monitoring of the human intestinal microbiota from the 2 nd week to 13 years of age. Anaerobe 28, 149-156 (2014).

55. Spor, A., Koren, O. \& Ley, R. Unravelling the effects of the environment and host genotype on the gut microbiome. Nat. Rev. Microbiol. 9, 279-290 (2011).

56. Sokol, H. et al. Faecalibacterium prausnitzii is an anti-inflammatory commensal bacterium identified by gut microbiota analysis of Crohn disease patients. Proc. Natl. Acad. Sci. USA 105, 16731-16736 (2008).

57. Kanai, T., Mikami, Y. \& Hayashi, A. A breakthrough in probiotics: Clostridium butyricum regulates gut homeostasis and anti-inflammatory response in inflammatory bowel disease. J. Gastroenterol. 50, 928-935 (2015).

58. He, B. et al. Intestinal bacteria trigger T cell-independent immunoglobulin A(2) class switching by inducing epithelial-cell secretion of the cytokine APRIL. Immunity 26, 812-826 (2007).

59. Xu, W. et al. Epithelial cells trigger frontline immunoglobulin class switching through a pathway regulated by the inhibitor SLPI. Nat. Immunol. 8, 294303 (2007).

60. Luoto, R., Kalliomaki, M., Laitinen, K. \& Isolauri, E. The impact of perinatal probiotic intervention on the development of overweight and obesity: follow-up study from birth to 10 years. Int. J. Obes. 34, 1531-1537 (2010).

61. Yan, F. \& Polk, D.B. Probiotic bacterium prevents cytokine-induced apoptosis in intestinal epithelial cells. J. Biol. Chem. 277, 50959-50965 (2002).

62. Caporaso, J.G. et al. QIIME allows analysis of high-throughput community sequencing data. Nat. Methods 7, 335-336 (2010).

63. Kennedy, R.J., Hoper, M., Deodhar, K., Erwin, P.J., Kirk, S.J. \& Gardiner, K.R. Interleukin 10-deficient colitis: new similarities to human inflammatory bowel disease. Br. J. Surg. 87, 1346-1351 (2000). 